\title{
Modeling survival at multi-population scales using mark-recapture data
}

\author{
V. Grosbois, ${ }^{1,2,8}$ M. P. Harris,${ }^{3}$ T. Anker-Nilssen, ${ }^{4}$ R. H. McCleery, ${ }^{5}$ D. N. Shaw, ${ }^{6}$ B. J. T. Morgan, ${ }^{7}$ \\ AND O. GIMENEZ ${ }^{1,7}$ \\ ${ }^{1}$ Laboratoire "Biométrie et Biologie Evolutive" UMR 5558, Bâtiment Gregor Mendel Université Claude Bernard Lyon 1, \\ 43 Boulevard du 11 Novembre 1918, 69622 Villeurbanne Cedex, France \\ ${ }^{2}$ Centre d'Ecologie Fonctionnelle et Evolutive, UMR 5175, 1919 Route de Mende, 34293, Montpellier Cedex 5, France \\ ${ }^{3}$ NERC Centre for Ecology and Hydrology, Edinburgh, Bush Estate, Penvick, Midlothian EH26 OQB United Kingdom \\ ${ }^{4}$ Norwegian Institute for Nature Research (NINA), NO-7485 Trondheim, Norway \\ ${ }^{5}$ Edward Grey Institute, Department of Zoology, University of Oxford, South Park Road, Oxford OX1 3PS United Kingdom \\ ${ }^{6}$ Fair Isle Bird Observatory Trust, Fair Isle, Shetland ZE29JU United Kingdom \\ ${ }^{7}$ Institute of Mathematics, Statistics and Actuarial Science, University of Kent, Canterbury, Kent CT2 $7 N F$ United Kingdom
}

\begin{abstract}
The demography of vertebrate populations is governed in part by processes operating at large spatial scales that have synchronizing effects on demographic parameters over large geographic areas, and in part, by local processes that generate fluctuations that are independent across populations. We describe a statistical model for the analysis of individual monitoring data at the multi-population scale that allows us to (1) split up temporal variation in survival into two components that account for these two types of processes and (2) evaluate the role of environmental factors in generating these two components. We derive from this model an index of synchrony among populations in the pattern of temporal variation in survival, and we evaluate the extent to which environmental factors contribute to synchronize or desynchronize survival variation among populations.

When applied to individual monitoring data from four colonies of the Atlantic Puffin (Fratercula arctica), 67\% of between-year variance in adult survival was accounted for by a global spatial-scale component, indicating substantial synchrony among colonies. Local sea surface temperature (SST) accounted for $40 \%$ of the global spatial-scale component but also for an equally large fraction of the local-scale component. SST thus acted at the same time as both a synchronizing and a desynchronizing agent. Between-year variation in adult survival not explained by the effect of local SST was as synchronized as total between-year variation, suggesting that other unknown environmental factors acted as synchronizing agents.

Our approach, which focuses on demographic mechanisms at the multi-population scale, ideally should be combined with investigations of population size time series in order to characterize thoroughly the processes that underlie patterns of multi-population dynamics and, ultimately, range dynamics.
\end{abstract}

Key words: Atlantic Puffin; Bayesian modeling; demography; deviance information criterion; environmental forcing; Fratercula arctica; mixed model; multi-population scale; sea surface temperature; synchronization; WinBUGS.

\section{INTRODUCTION}

Scaling up from single-population to multi-population dynamics is of basic importance to conservation ecology (Tilman and Kareiva 1997, Bascompte and Solé 1998, Koenig 1999) and, in particular, to our understanding of species range modifications under the influence of global changes (Parmesan and Yohe 2003, Sæther et al. 2003). Many empirical studies of time series of population abundance (reviewed in Liebhold et al.

Manuscript received 5 September 2008; revised 13 January 2009; accepted 21 January 2009. Corresponding Editor: M. Fortin.

8 Present address: CIRAD, UR AGIRs (Animal et Gestion Intégrée des Risques), TA C 22/E Campus International Baillarguet, Montpellier 34398 Cedex 5, France.

E-mail: vladimirgrosbois@hotmail.com
[2004], as well as elaborate theoretical and statistical models (reviewed in Ranta et al. [1997], Bjørnstad et al. [1999], Lundberg et al. [2000], and Liebhold et al. [2004]), have addressed the mechanisms behind multipopulation dynamics. It turns out that these mechanisms are difficult to infer via the sole analysis of population time series (Bjørnstad et al. 1999, Lande et al. 1999, Engen et al. 2002, Liebhold et al. 2004). More detailed empirical investigations are needed. Two obvious, but still poorly developed, approaches might be considered: the analysis of demographic parameters at multi-population spatial scales and the identification of environmental factors that influence multi-population dynamics.

The mechanisms behind spatiotemporal dynamics are interpreted most easily through studies of local demo- 
graphic rates because determinants of these dynamics ultimately exert their influence via local demographic rates (Bjørnstad et al. 1999). So far, few studies have investigated temporal variation in demographic parameters at the multi-population scale. One major reason for this relative scarcity is the difficulty of maintaining concurrent labor-intensive field studies for obtaining detailed information at the individual scale (Schwarz and Seber 1999, Pollock 2000). Although demographic studies at multi-population levels that often stem from collaborations involving several research teams have started to emerge (e.g., Paradis et al. 2000, Ringsby et al. 2002, Both et al. 2004, Frederiksen et al. 2005; see Grosbois et al. [2008] for a review of the studies on survival), statistical models designed specifically to address temporal variation at multi-population scales in demographic parameters and, in particular, in survival rates estimated through the analysis of markrecapture data, are poorly developed.

Although environmental factors are major determinants of multi-population dynamics (Moran 1953), there appear to be few published analyses of geographical synchrony in environmental factors, and even fewer studies of the similarities between such factors and the populations they are likely to influence (Koenig 1999; but see, e.g., Stenseth et al. 1999, Beaugrand and Reid 2003, Richardson and Schoeman 2004, Grøtan et al. 2005, Sæther et al. 2006). Statistical models for analyzing multi-population dynamics data that address explicitly and thoroughly the influences of environmental factors and their possible geographic variation have emerged only recently (Cazelles and Stone 2003, Engen and Sæther 2005). However, equivalents of these models for analyzing climate impacts on demographic parameters, particularly survival, based on multi-population individual monitoring data, to our knowledge are still lacking. Such statistical models are needed, particularly in order to obtain estimates for input parameters of predictive demographic models of the impact of climate change at the scale of distribution ranges (Sæther et al. 2003).

A few studies have addressed variation at the multipopulation scale in survival rates estimated through the analysis of mark-recapture data (reviewed in Grosbois et al. [2008]). However, because no statistical models designed specifically to address variation at multipopulation scales were available, neither the mechanisms underlying these variation nor the role of environmental factors in these mechanisms could be addressed rigorously. Here we introduce a mixed statistical model that addresses explicitly the temporal variation at the multi-population scale in survival rates estimated through the analysis of mark-recapture data. This model allows the splitting up of temporal variation in survival at the multi-population scale into a component that accounts for large-scale processes and a component that accounts for local-scale processes, with quantification of the contribution of environmental factors to these two components of variation. The influence of environmental covariates is accommodated by fixed effects. In addition, the model includes a random part that captures the variation in survival not explained by the covariate(s). This random part is structured into one global-scale component acting on all populations, and one local-scale component independent across populations. Because random effects are difficult to cope with in a classical maximum-likelihood framework (Burnham and White 2002), we employed a Bayesian approach using MCMC (Markov Chain Monte Carlo) simulations to estimate all of the model parameters. Based on the estimates obtained from the model that we introduce, we derive an index of synchrony among populations in the pattern of between-year variation in survival and we evaluate the extent to which environmental factors contribute to synchronize or desynchronize survival among populations.

We analyzed mark-recapture data of Atlantic Puffins (Fratercula arctica; see Plate 1) at four widely dispersed colonies in the northeast Atlantic for which Harris et al. (2005) reported an influence of sea surface temperature (SST) around the breeding colonies on adult survival. We asked whether local SSTs or other undetected environmental factors could account for the global-scale and the local-scale components of between-year variation in adult survival and, thereby, act as synchronizing or desynchronizing agents on survival over a large part of the latitudinal range of the species.

\section{Material and Methods}

We focused on the survival of individually marked adult Atlantic Puffins (hereafter puffins) monitored from 1990 to 2001 at four unconnected colonies in the east Atlantic: Skomer in Wales $\left(51^{\circ} 45^{\prime} \mathrm{N}, 5^{\circ} 18^{\prime} \mathrm{W}\right)$, Isle of May $\left(56^{\circ} 11^{\prime} \mathrm{N}, 2^{\circ} 34^{\prime} \mathrm{W}\right)$ and Fair Isle $\left(59^{\circ} 32^{\prime} \mathrm{N}\right.$, $1^{\circ} 38^{\prime} \mathrm{W}$ ) in Scotland, and Røst, one of the Lofoten Islands, Norway $\left(67^{\circ} 26^{\prime} \mathrm{N}, 11^{\circ} 52^{\prime} \mathrm{E}\right)$; see Fig. 1. In the previous study by Harris et al. (2005), a fifth colony, located on Hornøya, in northern Norway, was included in the analysis. We did not include Hornøya in the present analysis because initial captures of birds there were not evenly distributed over the study period (i.e., extremely high numbers of birds were captured for the first time in 1994 and 1995; see Harris et al. 2005: Appendix 1); we feared that such an irregular pattern of inclusion of newly marked individuals in the sample would result in confounding time and age effects. Details on the field protocols for the four other colonies can be found in Harris et al. (2005).

January to May mean SST was collected from ship, buoy, and bias-corrected satellite data at a resolution of $1^{\circ}$ latitude $\times 1^{\circ}$ longitude (available online). ${ }^{9}$ Mean SST in a sea area of $\sim 40000 \mathrm{~km}^{2}$ around each study colony

${ }^{9}$ 〈http://iridl.ldeo.columbia.edu/SOURCES/.IGOSS/. nmc/.Reyn_SmithOIv2/.monthly/.sst/> 


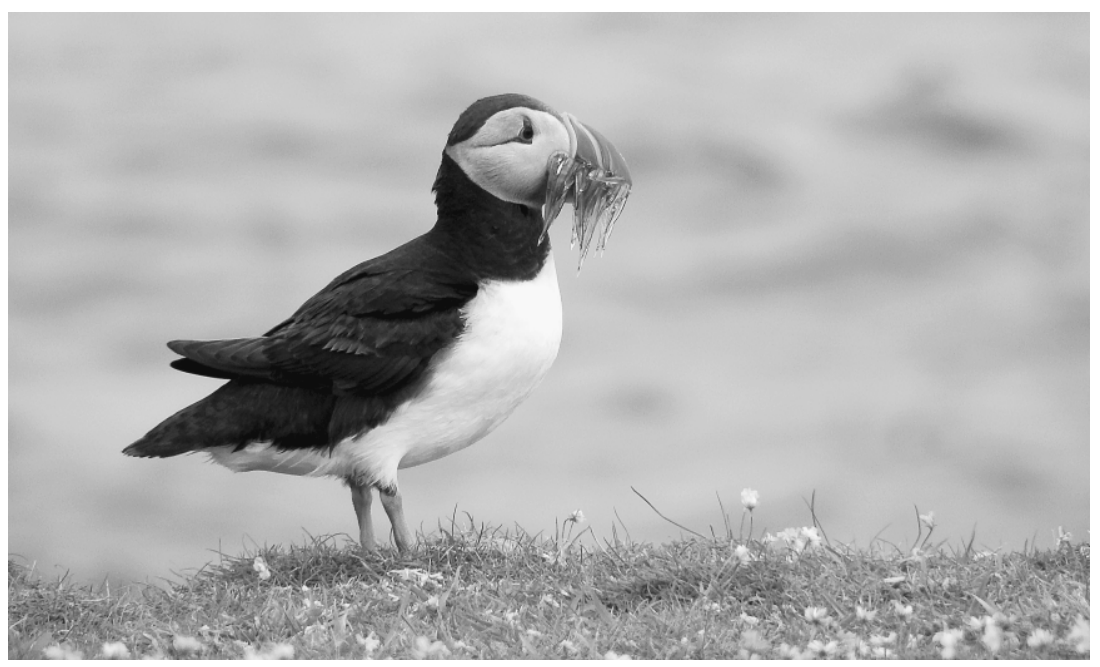

Plate 1. A puffin with a beakfull of sandeels on Skomer Island. Photo credit: D. N. Shaw.

was considered as an environmental covariate potentially influencing survival (Harris et al. 2005). For each colony, 6-10 cells of the grid were selected to represent an area of sea of $\sim 40000 \mathrm{~km}^{2}$ around the colony under study, and January to May SST was averaged over these cells. For each colony this covariate was then centered around the average and standardized by the standard deviation in the time series covering the study period. Strong positive correlation among local SST time series was found for five out of the six pairs of colonies (Pearson correlation coefficient $r>0.60$ ), whereas SST showed no correlation $(r=0.08)$ for the pair Skomer/Røst (Harris et al. 2005). SST was thus spatially autocorrelated at the scale covering the study colonies.

\section{A new statistical model for addressing variation in puffin survival at the multi-colony scale}

In a previous analysis of these data, Harris et al. (2005) used a deviance decomposition procedure to roughly evaluate the synchrony of interannual variation in survival among colonies. This method was a useful first step, but it was not accurate because it could not disentangle process variation and sampling variation (Burnham and White 2002). Here, we introduce mixedeffects structures (Dobson 2002) that allow the extraction of between-year variance in survival and estimation of its various components.

We consider standard models for mark-recapture data stratified by populations (Nichols et al. 1993). Under relevant hypotheses (e.g., Williams et al. 2002), the likelihood can be written as a product of multinomial distributions corresponding to the cells of the expected values of the number of released individuals, with which are associated probabilities that are complex nonlinear functions of both survival and capture probabilities. We consider the following regression model for the probability $\phi_{i s}$ that an individual survives from time $i$ to time $i+1(i=1, \ldots, I)$ in population $s(s=$ $1, \ldots, N)$ :

$$
\operatorname{logit}\left(\phi_{i s}\right)=\log \left(\frac{\phi_{i s}}{1-\phi_{i s}}\right)=f_{s}\left(x_{i s}\right)+\delta_{i}+\varepsilon_{i s}
$$

where $x_{i s}$ is the value of the covariate applying in population $s$ in the time interval from $i$ to $i+1$ (i.e., standardized local SST in January-May of year $i-1), f_{s}$ is a smooth function for population $s, \delta_{i}$ is a year random term, and $\varepsilon_{i s}$ is a year $\times$ population random term. The $\delta_{i}$ 's are independent, identically distributed random terms drawn at each occasion $i$ from a normal distribution: $\delta_{i} \sim N\left(0, \sigma_{\delta}^{2}\right)$. The $\varepsilon_{i s}$ 's are independent, identically distributed random terms drawn at each occasion $i$ and for each population $s$ from another normal distribution: $\varepsilon_{i s} \sim N\left(0, \sigma_{\varepsilon}^{2}\right)$. We also assume that the $\delta_{i}$ 's and the $\varepsilon_{i s}$ 's are independent from each other. In



FIG. 1. Geographic locations of four Atlantic Puffin (Fratercula arctica) colonies in the United Kingdom and Norway. 
the mixed model specified in Eq. 1, it is assumed that between-year variance in survival unexplained by the covariate $x$ equals $\sigma_{\delta}^{2}+\sigma_{\varepsilon}^{2}$ for all colonies, and that it is identically partitioned in all colonies into one globalscale component, $\sigma_{\delta}^{2}$, and one local-scale component, $\sigma_{\varepsilon}^{2}$. Note that this implies that the variance, $\sigma_{\varepsilon}^{2}$, of the year $\times$ population random term is identical in all populations. Finally it is also assumed that there is no correlation in survival between two dates $i$ and $j$ in the same colony as well as between colonies. Although this last assumption might be unrealistic if captures are performed on a weekly or monthly basis, we do not expect annual survival rates to be dependent from one year to the next. In addition, our data time series are too short to permit any autoregressive modeling (Johnson and Hoeting 2003).

The logit link used in Eq. 1 is one of several possible link functions that could be used. The function $f_{s}$ in Eq. 1 specifies a nonparametric flexible relationship between the survival probability and the covariate that allows nonlinear environmental trends to be detected (see Gimenez et al. [2006] and the Appendix for a more detailed description of this function). The difficulty here in estimating parameters is the presence of random effects. The frequentist approach would thus require maximizing the likelihood, which is obtained by integrating the likelihood over the random effects (e.g., Johnson and Hoeting 2003). This is therefore a problem involving a high-dimensional integral that could be handled by using approximations (Chavez-Demoulin 1999, Burnham and White 2002, Wintrebert et al. 2005). We preferred to adopt a Bayesian approach using MCMC sampling (Gilks et al. 1996). Summaries of the posterior distribution were obtained using Gibbs sampling in the software package WinBUGS (Spiegelhalter et al. 2003). Details regarding the Bayesian models and the MCMC procedure used to fit them are provided in the Appendix. The computer program used for fitting the model and the multi-colony puffin data are in the Supplement.

\section{Goodness-of-fit tests}

Using program U-CARE (Choquet et al. 2001), which implements specific contingency table procedures, we assessed the fit of the general time-dependent CormackJolly-Seber (CJS) model for each colony to determine whether it provided an adequate description of the data. We removed the first capture to limit heterogeneity among individuals. The CJS model fitted the data poorly for all colonies. A closer inspection revealed that the lack of fit of the CJS model was, to a large extent, accounted for by component 2CT, which detects heterogeneity in recapture probability (Isle of May: $\chi^{2}$ $=71.57, P<0.001$; Fair Isle: $\chi^{2}=254.18, P<0.001$; Skomer: $\chi^{2}=314.67, P<0.001$; Røst: $\chi^{2}=104.28, P<$ 0.001 ; $\mathrm{df}=10$ for all sites). This indicates "trap happiness," meaning that capture probability at year $i+1$ was higher for individuals captured at year $i$ than for individuals not captured at year $i$ (Pradel 1993). As advised by Pradel (1993), we incorporated an effect of time elapsed since last recapture in the modeling of recapture probability. This effect distinguishes between the two events that a capture occurred (capture probability denoted $p$ ) or not (capture probability denoted $p^{\prime}$ ) on the occasion before (Pradel 1993). Component 3SR, which detects heterogeneity in survival probability, was satisfactory except for Skomer (Isle of May: $\chi^{2}=14.72, \mathrm{df}=11, P=0.20$; Fair Isle: $\chi^{2}=13.11$, $\mathrm{df}=9, P=0.16$; Skomer: $\chi^{2}=38.70, \mathrm{df}=11, P<0.001$; Røst: $\left.\chi^{2}=11.34, \mathrm{df}=10, P=0.33\right)$.We considered that the slight over-dispersion generated by this heterogeneity in survival for Skomer was accounted for by the random terms in the models fitted.

\section{Reduced models and model selection}

In order to identify a model structure that is as parsimonious as possible while still describing the data satisfactorily, we fitted a set of reduced models for survival in which one or several of the three terms in the departure model defined by Eq. 1 (i.e., the relationship with the environmental covariate: $f(x)$, the year random term $\delta$, or the year $\times$ colony random term $\varepsilon$ ) were dropped. Note that in models where the relationship with the environmental covariate was dropped, a colony main effect that accounted for potential differences among colonies in baseline survival was kept in the fixed part of the model. In all models considered, recapture probability was considered as time- and colony-dependent and an effect of time elapsed since last recapture was also included.

To select between the models fitted, we used the deviance information criterion (DIC; Spiegelhalter et al. 2003), which can be seen as the AIC Bayesian counterpart for model selection. It is calculated as DIC $=-2 \log$ $\mathrm{L}($ data $\mid \theta)-2 \mathrm{pD}$, where $\theta$ denotes the model parameters, $\mathrm{L}($ data $\mid \theta)$ is the likelihood, and $\mathrm{pD}$ is the effective number of parameters. The model with the smallest DIC was selected as the best.

Statistics for assessing synchrony and contribution of environmental covariates in generating synchrony

Synchrony at the global scale was quantified based on the outputs of the models including the two random terms $\delta$ and $\varepsilon$ by calculating the intra-class correlation:

$$
\mathrm{ICC}=\frac{\sigma_{\delta}^{2}}{\sigma_{\delta}^{2}+\sigma_{\varepsilon}^{2}} .
$$

ICC quantifies the fraction of between-year variance unexplained by the environmental covariate or of total between-year variance (depending on whether the model does or does not include the relationship with the environmental covariate) accounted for by a global-scale component. When ICC is large, the global-scale variance component is large relative to the local-scale variance component. Between-year variation unexplained by the 
TABLE 1. Posterior medians (SD in parentheses) for the parameters of the model "parametric $+\delta$ " (with the lowest deviance information criterion; see Table 2) applied to the Atlantic Puffin (Fratercula arctica) data set.

\begin{tabular}{ccrrr}
\hline \hline Parameter & Skomer & Isle of May & Fair Isle & Røst \\
\hline$\phi$ & $0.90(0.01)$ & $0.94(0.01)$ & $0.87(0.02)$ & $0.89(0.01)$ \\
$p$ & $0.71(0.06)$ & $0.87(0.07)$ & $0.71(0.07)$ & $0.79(0.06)$ \\
$p^{\prime}$ & $0.61(0.06)$ & $0.82(0.07)$ & $2.22(0.10)$ & $0.71(0.08)$ \\
$\alpha_{0}$ & $2.32(0.15)$ & $-0.95(0.20)$ & $-0.46(0.15)$ & $2.50(0.16)$ \\
$\alpha_{1}$ & $-0.27(0.12)$ & $-0.09(0.11)$ & & $0.42(0.12)$ \\
$\alpha_{2}$ & & $-0.16(0.10)$ & \\
\hline
\end{tabular}

Notes: Parameters are: $\phi$, survival probability; $p$ and $p^{\prime}$, recapture probability for individuals recaptured and not recaptured a year before, respectively; $\alpha_{0}, \alpha_{1}, \alpha_{2}$, intercept, slope of the linear term, and slope of the quadratic term of the relationship between survival and SST, respectively. For survival and capture probabilities, geometric means of the year-specific estimates were computed. Due to a difference in the statistical procedure used to obtain them, the values presented here for mean survival rates differ slightly from the figures reported in Harris et al. (2005). The former are geometric means of annual estimates of survival from a time-dependent model, whereas the latter were directly obtained from models where survival was constrained to be constant in time. Across all four sites, the global-scale variance component was $\sigma_{\delta}^{2}=0.13(0.13)$. Blank cells in the last row indicate that $\alpha_{2}$ was not estimated for these three sites because the most relevant statistical models for these sites did not include this parameter.

environmental covariate or total between-year variation is then synchronous among colonies.

The contribution of the effect of local SST in generating synchrony was evaluated based on the outputs of the two models that included the two random terms $\delta$ and $\varepsilon$. One of these models (defined by Eq. 1) included the relationship with local SST, whereas in the other model this relationship had been dropped and only a colony main effect, which accounted for potential differences among colonies in baseline survival, was kept in the fixed part of the model. Let us define $\hat{\sigma}_{\delta}^{2}(\mathrm{res})$ and $\hat{\sigma}_{\delta}^{2}($ tot $)$ to be the estimates of global-scale between-year variance obtained in the models including and excluding the relationship with local SST, respectively. Let us also define $\hat{\sigma}_{\varepsilon}^{2}$ (res) and $\hat{\sigma}_{\varepsilon}^{2}$ (tot) as the estimates of local-scale between-year variance obtained in the models including and excluding the relationship with local SST, respectively. Following the method described in Franklin et al. (2000) and in Loison et al. (2002) for evaluating the contribution of the effect of an environmental covariate to between-year variance in survival, we used

$$
C_{\delta}=1-\frac{\hat{\sigma}_{\delta}^{2}(\text { residual })}{\hat{\sigma}_{\delta}^{2}(\text { total })}
$$

and

$$
C_{\varepsilon}=1-\frac{\hat{\sigma}_{\varepsilon}^{2}(\text { residual })}{\hat{\sigma}_{\varepsilon}^{2}(\text { total })}
$$

as measures of the contribution of local SST to globalscale and local-scale between-year variance, respectively.

\section{RESULTS}

Regarding the detection process, the geometric medians of capture probabilities are higher if a capture has occurred in the year before (Table 1), in agreement with a recent study on Atlantic Puffins (Harris et al. 2005). Annual survival rates through time are given in Fig. 2. We did not find any latitude gradient in mean survival (Table 1).
Whether the fixed part of the model included an effect of SST or not, models in which the random portion contained only a year effect (i.e., $\delta$ ) performed better in terms of DIC than models including in addition a year $\times$ colony effect (i.e., $\varepsilon$ ), and than models where no random effect was included (Table 2). Hence, total between-year variation and variation unexplained by the effect of SST were satisfactorily described by a global-scale component (Tables 1 and 2). Using models including both a year and a year $\times$ colony random terms, it was possible to evaluate ICC for total betweenyear variance (i.e., in a model that did not contain the effect of local SST) and for between-year variance unexplained by SST (i.e., in a model that did contain the effect of local SST). ICC took the same value for total and for unexplained variation, and $67 \%$ of the total and unexplained variation were accounted for by the global-scale variance component (Table 3). The between-year variation that remained unexplained after the effect of local SST had been accounted for was thus as synchronized as was total between-year variation.

Whatever the random part considered, models in which the fixed part contained the effect of local SST performed better in terms of DIC than did models in which this effect was not included (Table 2). The influence of increasing SST was positive for Atlantic Puffins on Røst but negative for the birds at two other colonies, Fair Isle and Skomer (Table 1, Fig. 3). Interestingly, the nonparameteric function reveals unexpected patterns in the survival function at Isle of May. Indeed, we found a quadratic relationship between survival and SST, suggesting that there is an optimum in survival at Isle of May around the intermediate SST values (Table 1, Fig. 3). Models in which the effect of local SST was accounted for through a parametric formulation performed better in terms of DIC than did models in which this effect was accounted for through a nonparametric formulation. However, we advocate the use of nonparametric functions as an exploratory step in the analysis, from which a parametric model can be 

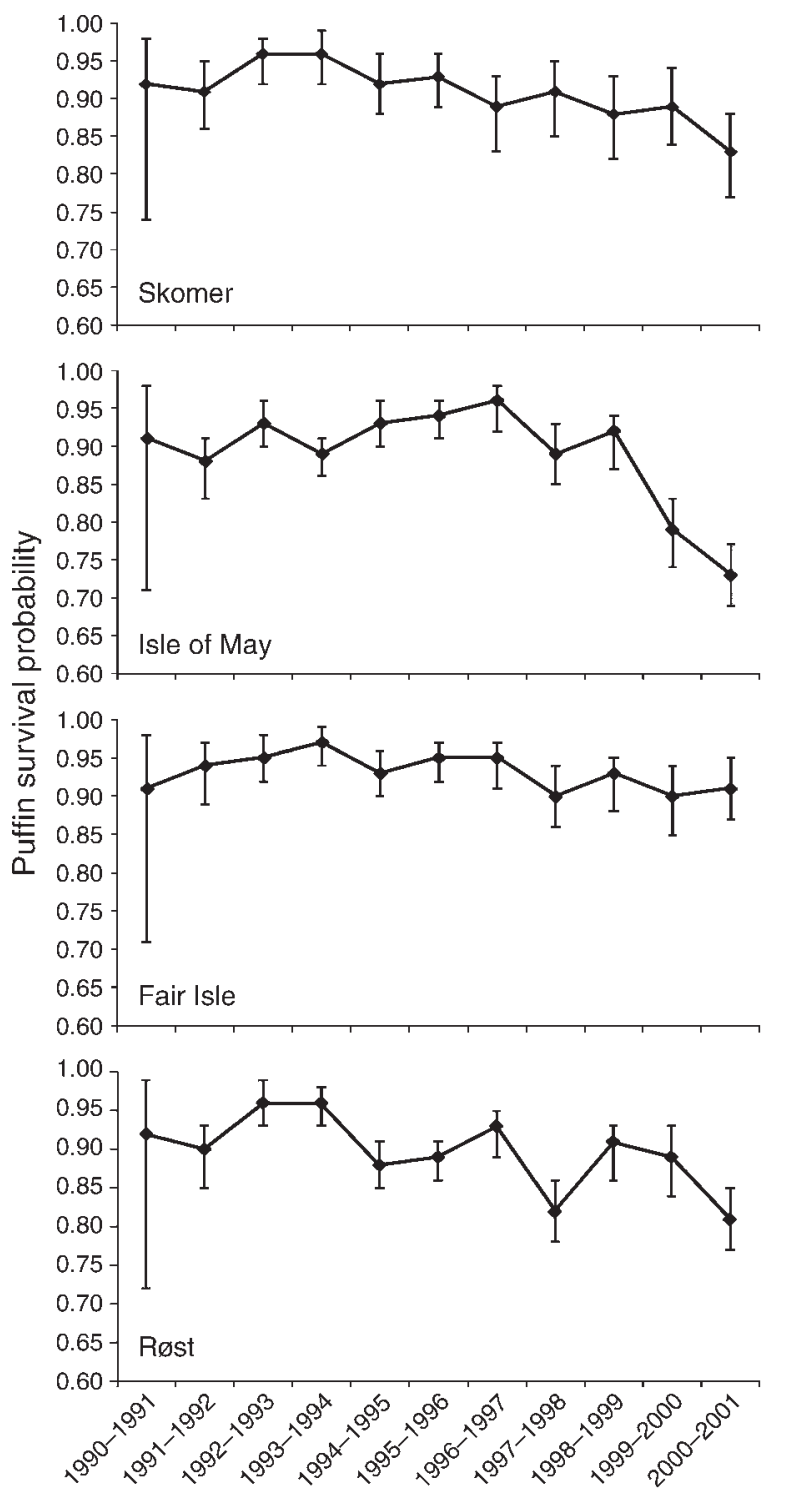

FIG. 2. Interannual fluctuations of survival probabilities in four Atlantic Puffin colonies from the eastern Atlantic (United Kingdom and Norway). Estimates of annual survival probabilities were obtained independently for each colony from a model with fully time-dependent survival. Vertical error bars represent the $95 \%$ pointwise credible intervals.

proposed as an alternative if desired (as for the quadratic relationship between local SST and survival for the Isle of May). The effect of local SST accounted for $39 \%$ of the global-scale component of between-year variance in survival (Table 3). In this respect, local SST acted as a synchronizing agent. However, local SST also accounted for $40 \%$ of the local-scale component of between-year variance in survival (Table 3), and thus acted also to some extent as a desynchronizing agent. Considering that local SST covaried positively among colonies, this last result was not expected. However, it is easily explained by the important variation detected among colonies in the sign and the shape of the relationships between local SST and adult survival.

\section{Discussion}

Recent analyses performed on time series of population size suggest that multi-population dynamics in vertebrates can be extremely complex. For instance, large-scale climatic factors or local climatic factors that are autocorrelated at large spatial scales can act on population dynamics as synchronizing agents at small spatial scales, and, at the same time as desynchronizing agents at large spatial scales, because their influence can vary geographically, sometimes being opposite in distant populations of the same species (Stenseth et al. 1999, Sæther et al. 2006). However, the mechanisms underlying such patterns are difficult to infer based solely on analysis of abundance time series because spatiotemporal variation in abundance results from complex demographic processes, implying several local demographic parameters that can be influenced similarly or differently by the same, or by different environmental factors and that can covary as a result of intrinsic constraints (cost of reproduction, density dependence). Furthermore, when the geographic range of the study is small enough and/or when the focal species is highly mobile, spatiotemporal dynamics are also governed by dispersal, the process that connects local populations through flows of individuals (Lebreton 1996, Lande et al. 1999, Paradis et al. 1999). Our approach focuses on the demographic mechanisms through which these multi-population patterns arise. It is complementary to, and should ideally be combined with, investigations of population size time series in order to characterize

Table 2. Models fitted to the Atlantic Puffin data.

\begin{tabular}{lcrc}
\hline \hline \multicolumn{1}{c}{ Model } & DIC & \multicolumn{1}{c}{ pD } & $\Delta$ DIC \\
\hline$f($ SST $)+\delta+\varepsilon$ & 8403.01 & 129.89 & 28.75 \\
$f($ SST $)+\varepsilon$ & 8405.44 & 134.68 & 31.18 \\
$f($ SST $)+\delta$ & 8387.05 & 105.00 & 12.79 \\
$f($ SST $)$ & 8403.73 & 96.56 & 29.47 \\
$\alpha+\delta+\varepsilon$ & 8419.38 & 145.74 & 45.12 \\
$\alpha+\varepsilon$ & 8408.82 & 139.68 & 34.56 \\
$\alpha+\delta$ & 8388.06 & 96.45 & 13.80 \\
$\alpha$ & 8455.31 & 91.13 & 81.05 \\
Parametric $+\delta+\varepsilon$ & 8389.12 & 119.26 & 14.86 \\
Parametric $+\varepsilon$ & 8396.92 & 127.83 & 22.66 \\
Parametric $+\delta$ & $\mathbf{8 3 7 4 . 2 6}$ & $\mathbf{9 6 . 0 8}$ & $\mathbf{0}$ \\
Parametric & 8405.13 & 90.23 & 30.87
\end{tabular}

Notes: DIC is the deviance information criterion, and $\mathrm{pD}$ is the number of effective parameters. $\triangle \mathrm{DIC}$ is the difference between the DIC of a model and the DIC for the minimum DIC model. For the models, $f(\mathrm{SST}), \alpha$, and parametric, respectively, denote a model with splines to model the effect of local SST (sea surface temperature) on survival, a model with a colony-specific mean effect only, and a model with a quadratic effect of local SST for Isle of May and a linear effect of local SST for all other colonies. In each of these models, we included both random terms $\delta$ (year random effect) and $\varepsilon$ (year $\times$ colony random effect), only one of them, or neither of them. The model achieving the best compromise between parsimony and fit is shown in boldface font. 
TABLE 3. Synchrony over four colonies of Atlantic Puffins, in total between-year variation in local adult survival and between-year variation unexplained by the effect of SST, and contribution of the effect of local SST to the global-scale $\left(C_{\delta}\right)$ and local-scale $\left(C_{\varepsilon}\right)$ variance components.

\begin{tabular}{lccc}
\hline \hline $\begin{array}{l}\text { Variance component } \\
\text { and synchrony }\end{array}$ & $\alpha+\delta+\varepsilon$ & Parametric $+\delta+\varepsilon$ & $\begin{array}{c}\text { Fraction of variation } \\
\text { accounted for by effect of SST }\end{array}$ \\
\cline { 2 - 3 } Global-scale $\left(\sigma_{\delta}^{2}\right)$ & $\hat{\sigma}_{\delta}^{2}($ total $)=0.18$ & $\hat{\sigma}_{\delta}^{2}($ residual $)=0.11$ & $C_{\delta}=1-\frac{\hat{\sigma}_{\delta}^{2}(\text { residual })}{\hat{\sigma}_{\delta}^{2}(\text { total })}=0.39$ \\
Local-scale $\left(\sigma_{\varepsilon}^{2}\right)$ & $\hat{\sigma}_{\varepsilon}^{2}$ (total $)=0.10$ & $\hat{\sigma}_{\varepsilon}^{2}($ residual $)=0.06$ & $C_{\varepsilon}=1-\frac{\hat{\sigma}_{\varepsilon}^{2}(\text { residual })}{\hat{\sigma}_{\varepsilon}^{2}(\text { total })}=0.40$ \\
ICC & 0.67 & 0.67 & \\
\hline
\end{tabular}

Notes: Synchrony is measured by ICC, the intra-class correlation coefficient. Global-scale and local-scale variance components are given before (total) and after (residual) accounting for the effect of sea surface temperature.

thoroughly the variations in the influence of climate factors according to geography and/or to habitat features. Such variations are of particular interest in the present climate change context because they imply that climate change could result in redistributions of populations at large (i.e., regime shifts) and local (i.e., habitat shifts) spatial scales.

\section{Influence of SST on spatiotemporal variation in adult survival of puffins}

The spatiotemporal variation in adult survival of the Atlantic Puffin seems, at first sight, to simply reveal a Moran effect on a demographic parameter. Adult survival was influenced by a local oceanographic covariate that covaried positively among colonies
(Harris et al. 2005). As a consequence, survival showed a substantial degree of synchronization among colonies (i.e., it was satisfactorily described by a global-scale variance component that accounted for $67 \%$ of betweenyear variation in all the colonies). However, as found recently in studies of population size time series of other vertebrates (Stenseth et al. 1999, Sæther et al. 2006), the potential for a spatially autocorrelated covariate such as local SST to synchronize adult survival variation was attenuated by geographical variation in the shape of its relationship with adult survival. Although adult survival decreased with increasing local SST in two colonies, the opposite pattern was detected at a third colony, and the relationship was quadratic in a fourth colony. We believe that, as a consequence, local SST acts as a

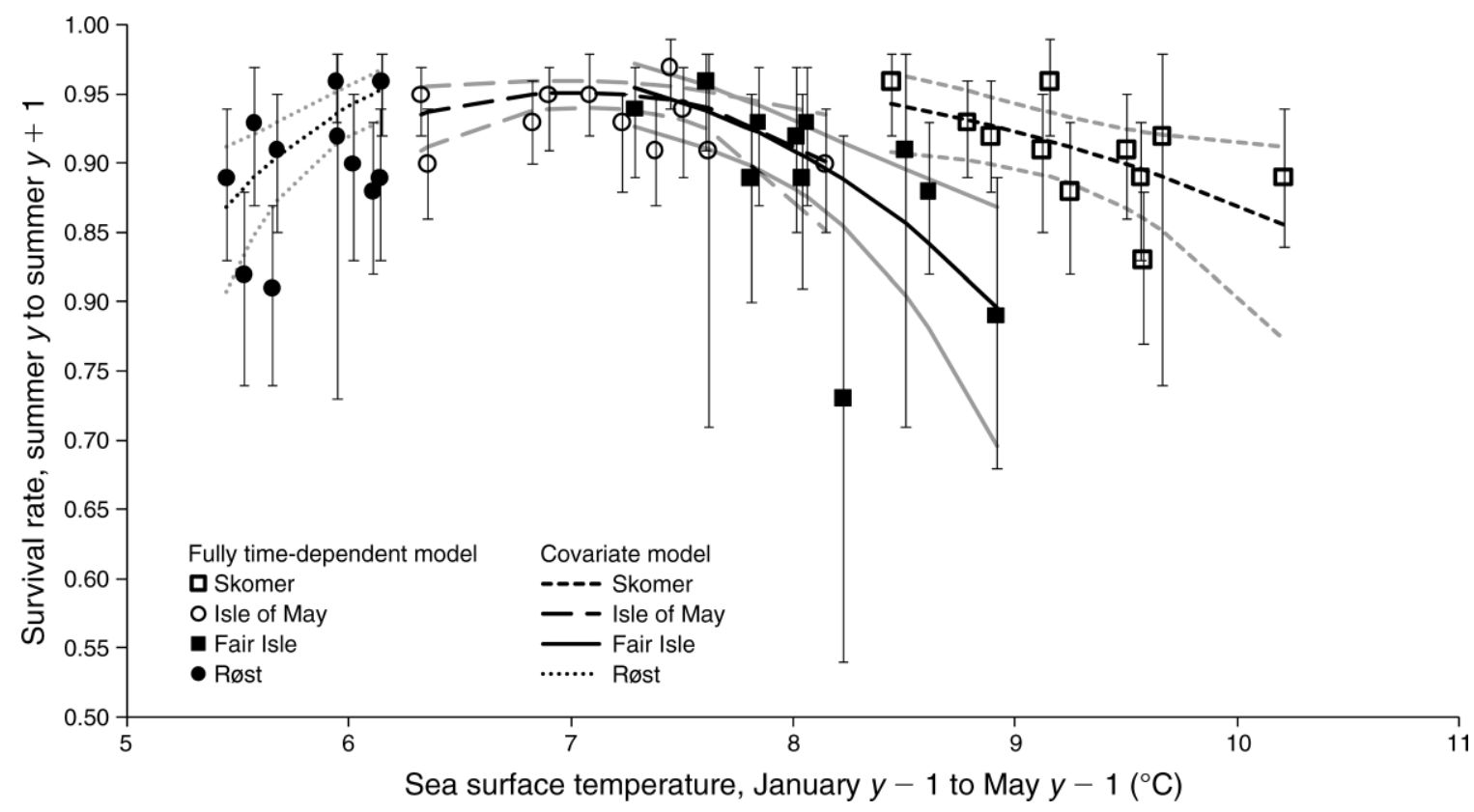

FIG. 3. Relationships between yearly survival of adult Atlantic Puffins and local sea surface temperature (SST) in four eastern Atlantic colonies. The estimates obtained from a fully time-dependent model are shown (symbols), with vertical error bars representing the $95 \%$ pointwise credible intervals. The estimates of the logit quadratic (for Isle of May) or logit linear (for the three other colonies) regression lines (black lines) were provided by the lowest DIC (deviance information criterion) model (see Tables 1 and 2) and are shown with $95 \%$ pointwise credible intervals (gray lines). 
synchronizing agent among most colonies but that it also resulted in desynchronizing temporal variation in survival, particularly in the colony of Røst. In accordance with this line of reasoning, we demonstrated that local SST accounted for as much as $40 \%$ of the globalscale component of between-year variation in adult survival, but also for an equally large fraction of its local-scale component.

We suggest two nonexclusive hypotheses for the mechanisms underlying the geographic variation in SST influence. The first hypothesis, already mentioned in Harris et al. (2005), states that local SST influences a compartment of the food chain upon which puffins rely, with geographic variation in specific composition: the forage fish compartment is dominated in the northernmost colony by a species (herring, Clupea harengus), the abundance of which increases with increasing SST (e.g., Sætre et al. 2002) and in the three other colonies by a species (sandeel, Amnodytes spp.), the abundance of which decreases with increasing SST (Arnott and Ruxton 2002). However, this interpretation fails to explain the quadratic relationship between SST and survival for the Isle of May, where sandeel is the main prey species. Under the second hypothesis, the relationship between SST and survival results from the influence of SST on primary production. Over a wide SST range encountered over the whole northeast Atlantic region, the relationship between primary production and SST is quadratic convex, with maximum productivity in intermediate SST regions (Richardson and Schoeman 2004). When more local, and thus narrower, SST ranges, such as those examined here for each colony, are considered, linear relationships can be detected with slopes that vary according to the relative position of the local range considered. A negative relationship is expected in regions where SST is relatively high (such as the regions where Fair Isle and Skomer are located), and a positive relationship in regions where SST is relatively low (the Røst region). In regions of intermediate SST (such as the region where the Isle of May colony is located) quadratic relationships between primary production and SST are expected. The quadratic relationship between SST and the survival of puffins breeding on the Isle of May is compatible with the hypothesis that the influence of SST on puffin survival results from that of SST on primary production over a vast area of the northeast Atlantic region. We could have precisely formalized this hypothesis with a model. For doing so, untransformed SST values, or SST values standardized using the mean and variance obtained for the full set of SST (i.e., the set including the SST time series for all the colonies) should have been used. Furthermore a unique, global quadratic relationship, applying to all four colonies, between the untransformed, or the globally standardized SST, and survival, should have been specified in the model rather than four independent relationships. However, it is obvious from Fig. 3, in which annual survival estimates obtained from a full time-dependent model and from the best model in the set that we considered (i.e., a model with one independent relationship with SST for each colony and a shared noise term) are plotted against untransformed values of the SST, that survival in Skomer is much higher than would be expected under the hypothesis of a global relationship between survival and SST at a large spatial scale encompassing the four colonies. Finally, neither of our two hypotheses concerning the biological processes underlying the relationship between survival and SST was fully supported by the results of the analysis.

Between-year variation in adult survival of puffins that remained unexplained after the effect of SST had been taken into account was as synchronized as was total between-year variation (i.e., $67 \%$ of it was accounted for by a global-scale variance component; Table 3). This novel result suggests that, in addition to SST in the vicinity of the breeding colonies, unknown environmental factors influence adult survival temporal variation in the puffin colonies and synchronize this variation among the colonies. Oceanographic, climatic, or trophic conditions in the wintering areas could be such factors because the wintering areas used by the birds of the different colonies considered here show substantial overlap (Harris et al. 2005). However, it is extremely difficult, if not impossible, to define and measure these factors because the distribution of puffins in winter is large and scattered (Harris et al. 2005) and because their diet during that time of year is largely unknown.

\section{Generalization to more complex demographic analyses}

Our current model applies to a relatively simple situation in which the populations under study are not connected by dispersal and where survival is the only demographic parameter in which spatiotemporal variation is addressed. Addressing spatiotemporal variation in other local demographic parameters that can be estimated through the analysis of CMR (capture-markrecapture) data should be straightforward using the model structure that we introduced. These include, for instance, the rate at which new breeders recruit into populations (Pradel 1996, Pradel and Lebreton 1999) or the proportion of the breeding populations that actually attempts to reproduce in intermittently breeding species (e.g., Jenouvrier et al. 2005).

An interesting perspective would be to generalize our model to simultaneous analyses of several local demographic parameters (e.g., survival and recruitment). CMR models that simultaneously address survival and recruitment do already exist (Pradel 1996). Because these models estimate for each time interval the rates at which individuals are gained (recruitment) and lost (mortality) in the population, they also produce estimates of the population growth rate (PGR) for each time interval (Pradel 1996). Another important generalization is necessary when sampling takes place over 
several connected sites. In these situations, multi-site mark-recapture models (Lebreton and Pradel 2002) allow the estimation of survival probabilities with possible discrepancies between locations, together with dispersal probabilities between sites (Sedinger et al. 2002, Hénaux et al. 2007). Adaptation of the mixedeffects structure introduced here to such models would allow estimating temporal covariances in local demographic processes among sites in addition to dispersal. The quantification of these two parameters is important to assess the dynamics of spatially structured populations (Lande et al. 1999).

\section{Generalization to more elaborate model structures}

In the formulation introduced so far, only one globalscale variance component, $\sigma_{\delta}^{2}$, and one local-scale variance component, $\sigma_{\varepsilon}^{2}$, were estimated. Note that with this basic formulation, correlations within pairs of populations are identical across pairs of populations. A promising generalization of our model would consist of specifying global- and local-scale variance components that are specific to each pair of populations. Such a generalization would allow modeling situations in which correlations vary among pairs of populations. In order to do so, we propose the following formulation:

$$
\begin{aligned}
\operatorname{logit}\left(\phi_{i s}\right) & =\log \left(\frac{\phi_{i s}}{1-\phi_{i s}}\right) \\
& =f_{s}\left(x_{i s}\right)+v_{s}\left[\sqrt{q_{s}} \delta_{i}+\sqrt{\left(1-q_{s}\right)} \varepsilon_{i s}\right] .
\end{aligned}
$$

In Eq. 2, $v_{s}$ is a measure of between-year variance for population $s ; Q_{i s}=\left[\sqrt{q_{s}} \delta_{i}+\sqrt{\left(1-q_{s}\right)} \varepsilon_{i s}\right]$ is a stochastic term that describes how between-year variance in population $s$ is partitioned between a global- and a local-scale components; $q_{s}$ is the fraction of betweenyear variance accounted for by the global-scale component (i.e., $\left.q_{s} \in[0,1]\right) ; \delta_{i} \sim N(0,1)$ is a random term representing the variation at the global scale; and $\varepsilon_{i s} \sim$ $N(0,1)$ is a random term representing the variation at the local scale. Assuming that $\sigma^{2}\left(\delta_{i}, \varepsilon_{i s}\right)=0$, one gets: $\sigma^{2}\left(Q_{i s}\right)=q_{s} \sigma^{2}\left(\delta_{i}\right)+\left(1-q_{s}\right) \sigma^{2}\left(\varepsilon_{i s}\right)$. Because $\sigma^{2}\left(\delta_{i}\right)=1$, and $\sigma^{2}\left(\varepsilon_{i s}\right)=1$, and $q_{s} \in[0,1]$, it follows that $\sigma^{2}\left(Q_{i s}\right)=1$. The between-year variance for colony $s$ is then $v_{s}^{2} \sigma^{2}\left(Q_{i s}\right)$ $=v_{s}^{2}$.

Based on Eq. 2, a model in which the fraction of between-year variance accounted for by a global-scale component is a function of a population-specific covariate (such as the distance between the focal population $s$ and all other populations $r: \Sigma_{r} d_{r s}$ ) is obtained by letting $q_{s}$ be a function of this covariate: $q_{s}=$ $f\left(\Sigma_{r} d_{r s}\right)$. This formulation would allow us to extend our approach to the geostatistical regression model framework (Cressie 1993, Diggle et al. 1998), which proposes, for example, that the correlation between any two populations is a function of the distance between them (Waller et al. 1997, Kleinschmidt et al. 2000). However, successfully fitting this type of model with empirical data would imply having access to capture-mark-recapture data sets with dozens of populations (B. P. Carlin, personal communication).

Finally, we are also currently developing generalizations of the model introduced here in which the fraction of between-year variance accounted for by a global-scale component is allowed to be structured by groups of populations. Such generalizations would allow us to address hypotheses such as stratification of the covariance among colonies according to diet similarities.

\section{Application to data sets where period of data availability overlaps only partially across populations}

In all the colonies considered here except that on Røst, individual monitoring data collected before 1990, and thus before the period considered here, were available (Harris et al. 2005). Thus, we could have constituted a data set comprising more study years for most colonies, but with periods for which data were available in the different colonies overlapping only partially rather than completely. No technical obstacle impedes the analysis, with the method we have introduced, of data from different populations over periods that overlap partially. It could further be argued that, for each population, considering the full period over which individual monitoring data are available allows one to maximize the number of statistical units for the analysis, and, thereby, to obtain more precise estimations of the parameters in the model. However, with this option, the weight of a given population in the estimation of the different variance components would be proportional to the length of the period of overlap with the other populations in the period over which data are available. As a consequence, the contribution to the estimation of the different variance components would be unbalanced among populations. Such an imbalance could bias the estimations of the variance components. This is the reason why, in our investigation of variation in puffin survival at the multi-population scale, we only considered the time period for which data were available in all the colonies (i.e., from 1990 to 2001).

\section{Conclusion}

Because of the complexity of the demographic processes involved, analyses of variation in time series of abundance and presence/absence at the multipopulation scale largely focus on the description of patterns (Bjørnstad et al. 1999, Lande et al. 2002). More process-oriented approaches unavoidably imply the analysis of individual-scale data that allow one to address variation in local demographic parameters at the multi-population scale and dispersal. This paper presents a Bayesian approach for modeling survival estimated from mark-recapture data at the multipopulation scale. We propose a way of splitting temporal variation in survival into global spatial scale and and local spatial scale components, quantifying synchrony of survival among populations, and assessing 
the role of environmental covariates in generating synchrony. Because of its potential for addressing demographic mechanisms at a large spatial scale, we believe that our approach can shed light on the processes that underlie patterns of multi-population dynamics and, ultimately, range dynamics.

\section{ACKNOWLEDGMENTS}

We thank the many people who helped collect the data used in these analyses. Studies on Skomer, Fair Isle, and the Isle of May were supported by the Joint Nature Conservancy Committee, and the one on Røst was supported by the Norwegian Directorate for Nature Management and the Environmental Protection Departments of the County Governors in Nordland, Troms, and Finnmark. We are grateful to four anonymous reviewers whose comments considerably improved the paper. V. Grosbois's research was supported the Centre National de la Recherche Scientifique (ACI CLIMPOP), the Ministère de l'Ecologie et du Développement Durable, and the Institut Français de la Biodiversite (program GICC2). O. Gimenez's research was supported by a MarieCurie Intra-European Fellowship within the Sixth European Community Framework Program. B. J. T. Morgan was supported by a Leverhulme Fellowship.

\section{Literature Cited}

Arnott, S. A., and G. Ruxton. 2002. Sandeel recruitment in the North Sea: demographic, climatic and trophic effects. Marine Ecology Progress Series 238:199-210.

Bascompte, J., and R. Solé. 1998. Modeling spatiotemporal dynamics in ecology. Springer-Verlag, New York, New York, USA.

Beaugrand, G., and P. C. Reid. 2003. Long-term changes in phytoplankton, zooplankton and salmon related to climate. Global Change Biology 9:801-817.

Bjørnstad, O. N., R. A. Ims, and X. Lambin. 1999. Spatial population dynamics: analyzing patterns and processes of population synchrony. Trends in Ecology and Evolution 14: 427-432.

Both, C., et al. 2004. Large-scale geographical variation confirms that climate change causes birds to lay earlier. Proceedings of the Royal Society of London B 271:16571662.

Burnham, K. P., and G. C. White. 2002. Evaluation of some random effects methodology applicable to bird ringing data. Journal of Applied Statistics 29:245-264.

Cazelles, B., and L. Stone. 2003. Detection of imperfect population synchrony in an uncertain world. Journal of Animal Ecology 72:953-968.

Chavez-Demoulin, V. 1999. Bayesian inference for smallsample capture-recapture data. Biometrics 55:727-731.

Choquet, R., A.-M. Reboulet, R. Pradel, and J.-D. Lebreton. 2001. U-care (Utilities: CApture-REcapture) user's guide. CEFE, CNRS, Montpellier, France.

Cressie, N. 1993. Statistics for spatial data. Wiley, New York, New York, USA.

Diggle, P. J., J. A. Tawn, and R. A. Moyeed. 1998. Modelbased geostatistics (with discussion). Applied Statistics 47: 299-350.

Dobson, A. J. 2002. An introduction to generalized linear models. Chapman and Hall, London, UK.

Engen, S., R. Lande, and B. E. Sæther. 2002. Migration and spatiotemporal variation in population dynamics in a heterogeneous environment. Ecology 83:570-579.

Engen, S., and B. E. Sæther. 2005. Generalizations of the Moran effect explaining spatial synchrony in population fluctuations. American Naturalist 166:603-612.

Franklin, A. B., D. R. Anderson, R. J. Gutiérrez, and K. P. Burnham. 2000. Climate, habitat quality, and fitness in
Northern Spotted Owl populations in northwestern California. Ecological Monographs 70:539-590.

Frederiksen, M., P. J. Wright, M. P. Harris, R. A. Mavor, M. Heubeck, and S. Wanless. 2005. Regional patterns of kittiwake Rissa tridactyla breeding success are related to variability in sandeel recruitment. Marine Ecology Progress Series 300:201-211.

Gilks, W. R., S. Richardson, and D. J. Spiegelhalter. 1996. Markov chain Monte Carlo in practice. Chapman and Hall, London, UK.

Gimenez, O., C. Crainiceanu, C. Barbraud, S. Jenouvrier, and B. J. T. Morgan. 2006. Semiparametric regression in capturerecapture modeling. Biometrics 62:691-698.

Grosbois, V., O. Gimenez, J.-M. Gaillard, R. Pradel, C. Barbraud, J. Clobert, A. P. Møller, and H. Weimerskirch. 2008. Assessing the impact of climate variation on survival in vertebrate populations. Biological Reviews 83:357-399.

Grøtan, V., B. E. Sæther, S. Engen, E. J. Solberg, J. D. C. Linnell, R. Andersen, H. Brøseth, and E. Lund. 2005. Climate causes large-scale spatial synchrony in population fluctuations of a temperate herbivore. Ecology 86:1472-1482.

Harris, M. P., T. Anker-Nilssen, R. H. McCleery, K. E. Erikstad, D. N. Shaw, and V. Grosbois. 2005. Effect of wintering area and climate on the survival of adult Atlantic puffins Fratercula arctica in the eastern Atlantic. Marine Ecology Progress Series 297:283-296.

Hénaux, V., T. Bregnballe, and J.-D. Lebreton. 2007. Dispersal and recruitment during population growth in a colonial bird, the great cormorant Phalacrocorax carbo sinensis. Journal of Avian Biology 38:44-57.

Jenouvrier, S., C. Barbraud, B. Cazelles, and H. Weimerskirch. 2005. Modelling population dynamics of seabirds: importance of the effects of climate fluctuations on breeding proportions. Oikos 108:511-522.

Johnson, D. S., and J. A. Hoeting. 2003. Autoregressive models for capture-recapture data: a Bayesian approach. Biometrics 59:341-350.

Kleinschmidt, I., M. Bagayoko, G. P. Clarke, M. Craig, and D. Le Sueur. 2000. A spatial statistical approach to malaria mapping. International Journal of Epidemiology 29:355-361.

Koenig, W. D. 1999. Spatial autocorrelation of ecological phenomena. Trends in Ecology and Evolution 14:22-26.

Lande, R., S. Engen, and B.-E. Sæther. 1999. Spatial scale of population synchrony: environmental correlation versus dispersal and density regulation. American Naturalist 154: 271-281.

Lande, R., S. Engen, B.-E. Sæther, F. Filli, E. Matthysen, and H. Weimerskirch. 2002. Estimating density dependence from population time series using demographic theory and lifehistory data. American Naturalist 159:321-337.

Lebreton, J.-D. 1996. Demographic models for subdivided populations: the renewal equation approach. Theoretical Population Biology 49:291-313.

Lebreton, J.-D., and R. Pradel. 2002. Multistate recapture models: modelling incomplete individual histories. Journal of Applied Statistics 29:353-369.

Liebhold, A., W. D. Koenig, and N. Bjørnstad. 2004. Spatial synchrony in population dynamics. Annual Review of Ecology, Evolution and Systematics 35:467-490.

Loison, A., B.-E. Sæther, K. Jerstad, and O. W. Røstad. 2002. Disentangling the sources of variation in the survival of the European dipper. Journal of Applied Statistics 29:289-304.

Lundberg, P., E. Ranta, J. Ripa, and V. Kaitala. 2000. Population variability in space and time. Trends in Ecology and Evolution 15:460-464.

Moran, P. A. P. 1953. The statistical analysis of the Canadian lynx cycle. 2. Synchronization and meteorology. Australian Journal of Zoology 1:291-298.

Nichols, J. D., C. Brownie, J. Hines, E., K. H. Pollock, and J. B. Hestbeck. 1993. The estimation of exchanges among populations or subpopulations. Pages 265-279 in J.-D. 
Lebreton and P. M. North, editors. Marked individuals in the study of bird population. Birkhäuser, Basel, Switzerland.

Paradis, E., S. R. Baillie, W. J. Sutherland, C. Dudley, H. Q. P. Crick, and R. D. Gregory. 2000. Large-scale spatial variation in the breeding performance of song thrushes Turdus philomelos and blackbirds T. merula in Britain. Journal of Applied Ecology 37:73-87.

Paradis, E., S. R. Baillie, W. J. Sutherland, and R. D. Gregory. 1999. Dispersal and spatial scale affect synchrony in spatial population dynamics. Ecology Letters 2:114-120.

Parmesan, C., and G. Yohe. 2003. A globally coherent fingerprint of climate change impacts across natural systems. Nature 421:37-42.

Pollock, K. H. 2000. Capture-recapture models. Journal of the American Statistical Association 95:293-296.

Pradel, R. 1993. Flexibility in survival analysis from recapture data: handling trap-dependence. Pages 29-37 in J.-D. Lebreton and P. M. North, editors. Marked individuals in the study of bird population. Birkhäuser, Basel, Switzerland.

Pradel, R. 1996. Utilization of capture-mark-recapture for the study of recruitment and population growth rate. Biometrics 52:703-709.

Pradel, R., and J.-D. Lebreton. 1999. Comparison of different approaches to the study of local recruitment of breeders. Bird Study 46:74-81.

Ranta, E., K. Veijo, J. Lindström, and E. Helle. 1997. The Moran effect synchrony in population dynamics. Oikos 78 : 136-142.

Richardson, A. J., and D. S. Schoeman. 2004. Climate impact on plankton ecosystems in the Northeast Atlantic. Science 305:1609-1612.

Ringsby, T. H., B.-E. Sæther, J. Tufto, H. Jensen, and E. J. Solberg. 2002. Asynchronous spatiotemporal demography of a House Sparrow metapopulation in a correlated environment. Ecology 83:561-569.

Sæther, B.-E., et al. 2003. Climate variation and regional gradients in population dynamics of two hole-nesting passerines. Proceedings of the Royal Society of London B 270:2397-2404.
Sæther, B.-E., V. Grøtan, P. Tryjanowski, C. Barbaud, S. Engen, and M. Fulin. 2006. Climate and spatio-temporal variation in the population dynamics of long distance migrant, the white stork. Journal of Animal Ecology 75: $80-90$.

Sætre, R., R. Torensen, and T. Anker-Nilssen. 2002. Factors affecting the recruitment variability of the Norwegian springspawning herring (Clupea harengus L.). ICES Journal of Marine Science 59:725-736.

Schwarz, C. J., and G. A. F. Seber. 1999. Estimating animal abundance: Review III. Statistical Science 14:427-456.

Sedinger, J. S., N. D. Chelgren, M. S. Lindberg, T. Obritchkewitch, M. T. Kirk, P. Martin, B. A. Anderson, and D. H. Ward. 2002. Life-history implications of largescale spatial variation in adult survival of Black Brant (Branta bernicla nigricans). Auk 119:510-515.

Spiegelhalter, D. J., A. Thomas, N. G. Best, and D. Lunn. 2003. WinBUGS user manual. Version 1.4. MRC [Medical Research Council] Biostatistics Unit, Cambridge, UK. $\langle$ www.mrc-bsu.cam.ac.uk/bugs/〉

Stenseth, N. C., K.-S. Chan, H. Tong, R. Boonstra, S. Boutin, C. J. Krebs, E. Post, M. O'Donoghue, N. G. Yoccoz, M. C. Forchhammer, and J. W. Hurrel. 1999. Common dynamic structure of Canada lynx populations within three climatic regions. Science 285:1071-1073.

Tilman, D., and P. Kareiva. 1997. Spatial ecology: the role of space in population dynamics and interspecific interactions. Princeton University Press, Princeton, New Jersey, USA.

Waller, L. A., B. P. Carlin, H. Xia, and A. E. Gelfand. 1997. Hierarchical spatio-temporal mapping of disease rates. Journal of the American Statistical Association 92:607-617.

Williams, B. K., J. D. Nichols, and M. J. Conroy. 2002. Analysis and management of animal populations. Academic Press, San Diego, California, USA.

Wintrebert, C. M. A., A. H. Zwinderman, E. Cam, R. Pradel, and J. C. van Houwelingen. 2005. Joint modelling of breeding and survival in the kittiwake using frailty models. Ecological Modelling 181:203-213.

\section{APPENDIX}

Detail of the modeling procedure (Ecological Archives E090-208-A1).

\section{SUPPLEMENT}

Scripts and multi-colony Atlantic Puffin data for running the mixed model for survival at the multi-population scale (Ecological Archives E090-208-S1). 